\title{
Proposal and Performance Evaluation of a Hybrid BPSK-Modified MPPM Technique for Optical Fiber Communications Systems
}

\author{
Hossam Selmy, Member, IEEE, Hossam M. H. Shalaby, Senior Member, IEEE, \\ and Zen-Ichiro Kawasaki, Senior Member, IEEE
}

\begin{abstract}
A hybrid binary phase shift keying-modified multipulse pulse position modulation (hybrid BPSK-modified MPPM) scheme is proposed as a new modulation technique to improve the performance of both conventional binary phase shift keying (BPSK) and multi-pulse pulse position modulation (MPPM) techniques in optical fiber communications systems. In conventional BPSK scheme, a consecutive stream of low power BPSK symbols are transmitted. However, in the proposed scheme, a less number of high power BPSK symbols are transmitted in a hybrid frame and their positions are exploited to transmit more bits. That is, the transmitted information is carried in both the positions and phases of the transmitted pulses. The transmission characteristics, transmitter and receiver block diagram, bandwidth-utilization, and optimum decoding process for the proposed scheme are all studied in this paper. Several performance measures are also derived and compared to those of conventional schemes in optical fiber channels. Our results reveal that, under an average power constraint, the proposed hybrid BPSK-modified MPPM scheme achieves much lower levels of bit-error rates and symbol-error rates than those of ordinary BPSK and ordinary MPPM schemes, respectively, for both moderate or high signal-to-noise ratios. In addition, the proposed modulation scheme achieves much higher bandwidth-utilization efficiencies than those of ordinary schemes.
\end{abstract}

Index Terms-Binary phase shift keying modulation (BPSK), bit-error rate, hybrid binary phase shift keying-modified multipulse pulse-position modulation (hybrid BPSK-modified MPPM), multi-pulse pulse position modulation (MPPM), optical fiber communications, symbol-error rate.

\section{INTRODUCTION}

$\mathbf{P}$ HASE modulations represent important modulation category for both optical fiber and free-space optical (FSO) communications. Coherent detection of phase modulated signals increases the receiver sensitivity over traditional direct detection of intensity modulated signals. For both homodyne

Manuscript received March 30, 2013; revised July 24, 2013 and September 3, 2013; accepted September 4, 2013. Date of publication October 3, 2013; date of current version October 25, 2013.

H. Selmy is with the Department of Electronics and Communications Engineering, Egypt-Japan University of Science and Technology (E-JUST), Alexandria 21934, Egypt (e-mail: hossamselmy@yahoo.com).

H. M. H. Shalaby is with the Department of Electronics and Communications Engineering, Egypt-Japan University of Science and Technology (E-JUST), Alexandria 21934, Egypt, on leave from the Electrical Engineering Department, Alexandria University, Alexandria 21544, Egypt (e-mail: shalaby@ieee.org).

Z.-I. Kawasaki is with the Graduate School of Engineering, Osaka University, Osaka 565-0871, Japan (e-mail: zen@comm.eng.osaka-u.ac.jp).

Digital Object Identifier 10.1109/JLT.2013.2284605 and heterodyne receivers, noticeable enhancements in the receiver sensitivity are achieved for systems limited by amplifier noise [1]. However, coherent detection of phase modulated signals requires much more complex receiver than the one used for direct detection of intensity modulated signals.

Pulse-position modulation techniques represent another important modulation category in optical communications [2]. One important format of this category is multi-pulse pulse-position modulation (MPPM) scheme, which has been proposed to enhance the bandwidth utilization efficiency of ordinary singlepulse pulse position modulation (PPM) scheme [3], [4]. Recently, multilevel pulse-position modulation for optical powerefficient communication has been proposed [5]. Generally, both PPM and MPPM have been used extensively with intensity modulation/direct detection (IM/DD) systems. However, for better receiver sensitivity, coherent detection has been recently proposed (including both homodyne and heterodyne detections) to demodulate such schemes [6], [7]. For shot-noise limited systems, the sensitivity of heterodyne detection receivers is better by $3 \mathrm{~dB}$ than that of the direct detection receivers.

For further enhancement in the performance of both phase modulations and pulse-position modulations, hybrid modulations are addressed. One direction is to combine phase modulations with pulse-position modulations in order to exploit the advantages of both schemes. The main advantage of using phase modulation is to increase the bandwidth utilization efficiency of the hybrid scheme. By combing phase modulation with pulseposition modulation, the average transmitted power can be further reduced, resulting in relatively high bandwidth-utilization efficiency at a reasonable receiver sensitivity.

The idea of hybrid phase and pulse-position modulations for optical communications has been recently proposed in many literature. Both classical and quantum receivers for hybrid BPSK2PPM scheme have been investigated and their performances have been compared to each other [8]. Another hybrid modulation, which combines both PPM and binary phase shift keying subcarrier intensity modulation (BPSK-SIM) has been proposed in [9]. This scheme offers BER performance that is superior to that of both BPSK-SIM and PPM at the same average transmitted power and bit rate. Recently, an experimental work has been carried out to demonstrate a record sensitivity in an optically pre-amplified receiver by combining both polarization-divisionmultiplexed quadrature phase-shift keying (PDM-QPSK) and M-ary pulse-position modulation [10]. Another experimental work is also demonstrated for long-haul transmission of 
PM-2PPM-QPSK at $42.8 \mathrm{Gbit} / \mathrm{s}$ [11]. Furthermore, the hybridization of $\mathrm{M}$-ary pulse-position modulation with frequencyshift keying is recently demonstrated for high-sensitivity optical transmission [12].

In this paper, we aim at enhancing the performance of both ordinary BPSK and MPPM modulations in optical fiber communications by proposing a new hybrid modulation scheme. Specifically, we propose a modified version of MPPM and modulate its signal pulses by ordinary BPSK modulation. We call the proposed modulation scheme hybrid binary phase shift keyingmodified MPPM (hybrid BPSK-modified MPPM). In the proposed scheme, information bits are encoded in both the phase and the positions of the transmitted pulses. Also, as the demodulation of these BPSK pulses requires the implementation of coherent detection (homodyne or heterodyne), we propose to use coherent detection for demodulating both BPSK and MPPM symbols in the hybrid frame.

The rest of the paper is organized as follows: In Section II, we develop a general system model for the hybrid BPSKmodified MPPM scheme and study its transmission characteristics. Also, the differences between the proposed hybrid scheme and the ordinary BPSK scheme are stated. In Section III, we present the transmitter and receiver block diagrams for the hybrid scheme. Also, we state its maximum-likelihood decoding (optimal decoding) in optically pre-amplified channels with amplifier-noise limited case. The enhancements in the ordinary BPSK and ordinary MPPM are investigated in Sections IV and V, respectively. Finally, the conclusion is given in Section VI.

\section{Proposed System Model AND CHARACTERISTICS}

Here, we propose a hybrid BPSK-modified MPPM modulation scheme in which binary phase shift keying is combined with a modified version of MPPM. The goal of proposing this hybrid scheme is to enhance the performance of both ordinary BPSK and ordinary MPPM modulation schemes used for optical communications systems. Comparing with BPSK, instead of transmitting a consecutive stream of BPSK pulses (each with a relatively small power), we transmit less number of high power BPSK pulses and exploit the positions of these pulses to transmit more bits in the hybrid frame. Precisely, with frames of size $M$ slots, instead of transmitting $M$ BPSK pulses in the ordinary scheme, $n_{H}$ modulated optical pulses are transmitted in the proposed hybrid frame. Also, comparing with ordinary MPPM, for frames of size $M$ slots, instead of simply transmitting $n_{M}$ un-modulated optical pulses in the ordinary MPPM frame, BPSK is used to modulate $n_{H}$ optical pulses in the hybrid frame. The frame structures of ordinary BPSK, proposed hybrid BPSK-modified MPPM scheme and coherent detection $n$-pulse MPPM scheme are explained in Figs. 1-3, respectively.

The term "modified MPPM" comes from the ability to increase the number of transmitted optical pulses per hybrid frame to values more than $M / 2$. This is in contrast to ordinary MPPM in which the maximum number of transmitted optical pulses per frame is limited to $M / 2$ as $\left(\begin{array}{c}M \\ n\end{array}\right)$ and $\left(\begin{array}{c}M \\ M-n\end{array}\right)$ have the same value [13].

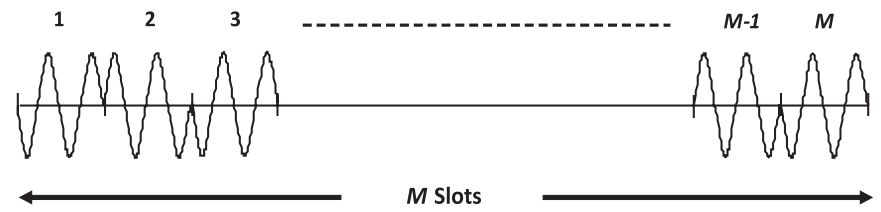

Fig. 1. $M$ consecutive symbols in ordinary BPSK scheme.

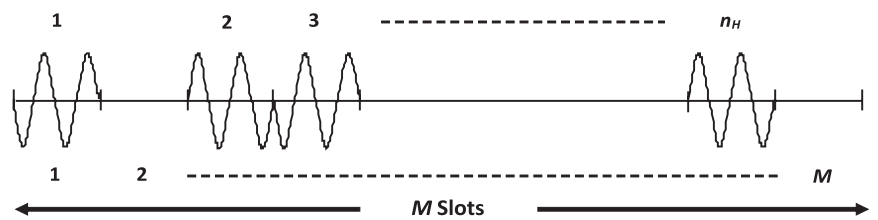

Fig. 2. Frame structure of a hybrid BPSK-modified MPPM scheme

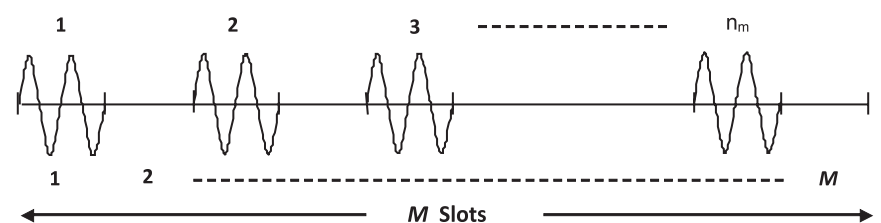

Fig. 3. Frame structure of a coherent detection $n$-pulse MPPM scheme.

\section{A. A Comparison With Ordinary BPSK}

Here, the number of transmitted bits for $M$ consecutive BPSK symbols is $M$ bits, whereas for hybrid BPSK-modified MPPM, this number is $n_{H}+\log _{2}\left(\begin{array}{c}M \\ n_{H}\end{array}\right)$ bits. To conserve the same transmission bits per frame for both schemes, there is a minimum value of $n_{H}$ that results in near or slightly larger transmission rate for hybrid one. In other words, for any value of $n_{H}$, the maximum frame size $M_{\max }$ that makes the transmission rate for hybrid scheme the same or slightly larger than that of the ordinary BPSK scheme is given by

$$
n_{H}+\log _{2}\left(\begin{array}{c}
M_{\max } \\
n_{H}
\end{array}\right) \geqslant M_{\max }
$$

Clearly, if we use frame sizes larger than $M_{\max }$, the hybrid frame would transmit less bits than that of the ordinary BPSK. Also, the percentage of maximum increase in pulse power for hybrid scheme under average power constraint is $\left(M_{\max }-n_{H}\right) / n_{H} \times 100 \%$. The values of $M_{\max }$ are plotted in the Fig. 4. It can be seen from the figure that for $n_{H}=3$ pulses, the maximum hybrid frame size that achieves almost same transmission rate of ordinary BPSK is 9 slots. In this case, the hybrid scheme transmits 9.1 bits per frame (slightly larger than ordinary BPSK transmission rate). With $M_{\max }=9$ slots, the percentage of maximum increase in pulse power under average power constraint is $200 \%$. It should be noticed that higher transmission rates for the hybrid scheme can be obtained by implementing less frame sizes. In this case the increase in pulse power would reduce accordingly.

Moreover, the bandwidth-utilization efficiency of the ordinary BPSK is further enhanced using the proposed hybrid scheme. For a hybrid scheme with frames of size $M$ slots, 


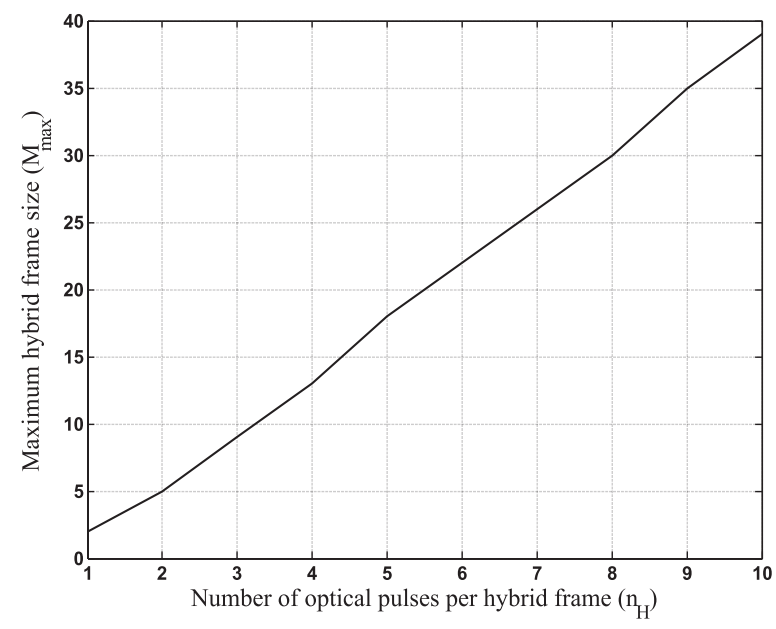

Fig. 4. The maximum hybrid frame size at the rate of ordinary BPSK.

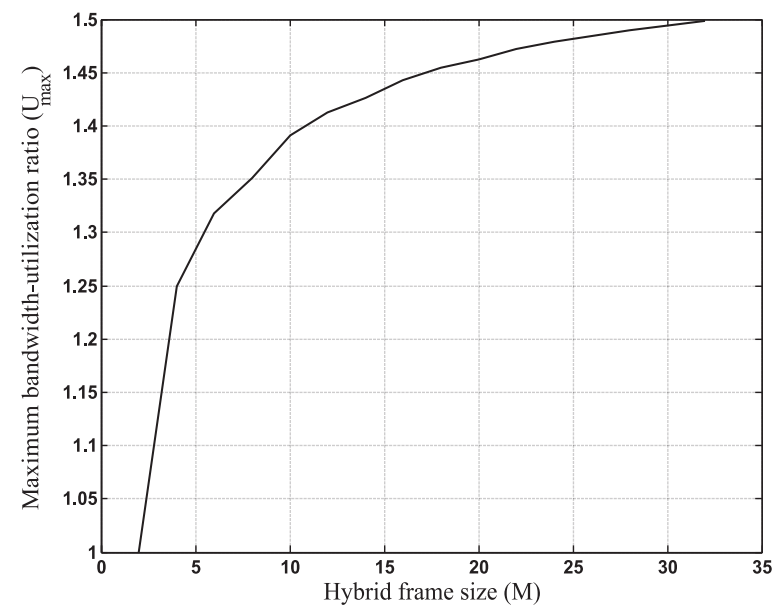

Fig. 5. Maximum bandwidth-utilization ratio $\left(U_{\max }\right)$.

each containing $n_{H}$ optical pulses, we define the maximum bandwidth-utilization ratio between the hybrid and the ordinary schemes $U_{\max }$ as

$$
U_{\max }=\max _{n_{H}} \frac{n_{H}+\log _{2}\left(\begin{array}{c}
M \\
n_{H}
\end{array}\right)}{M} .
$$

The maximum bandwidth-utilization ratio versus the hybrid frame size $M$ is indicated in Fig. 5. Clearly, $U_{\max }$ increases gradually with increasing hybrid frame size until reaching its saturation at values of $M$ larger than 20 slots.

\section{B. A Comparison With Ordinary MPPM}

Here, the number of transmitted bits per frame for ordinary MPPM is $\log _{2}\left(\begin{array}{c}M \\ n_{M}\end{array}\right)$ bits, whereas for the proposed hybrid BPSK-modified MPPM scheme, this number is $n_{H}+\log _{2}\left(\begin{array}{c}M \\ n_{H}\end{array}\right)$ bits. Therefore, the bandwidth-utilization efficiency for the ordinary MPPM scheme is given by

$$
U_{M}=\frac{\log _{2}\left(\begin{array}{c}
M \\
n_{M}
\end{array}\right)}{M} .
$$

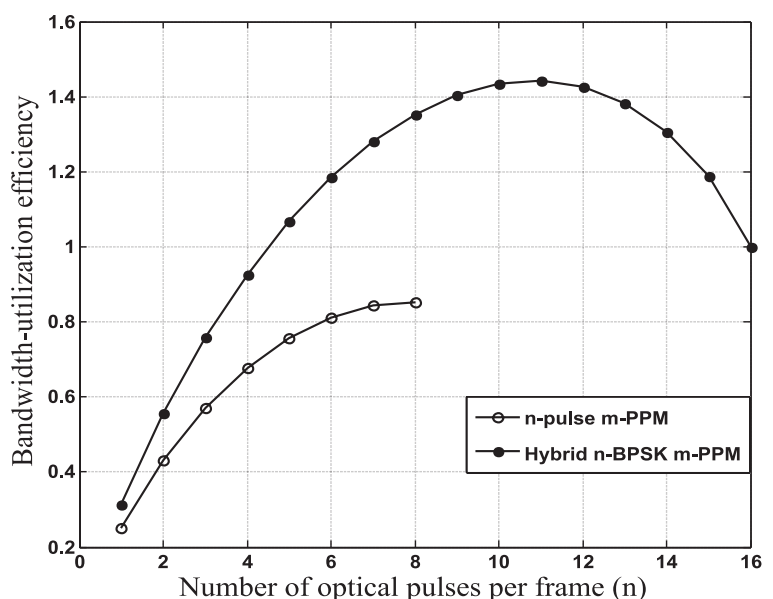

Fig. 6. Bandwidth utilization efficiency.

Whereas for the hybrid BPSK-modified MPPM scheme, it is given by

$$
U_{H}=\frac{n_{H}+\log _{2}\left(\begin{array}{c}
M \\
n_{H}
\end{array}\right)}{M} .
$$

The bandwidth-utilization efficiencies for both schemes are compared at a fixed frame size of 16 slots and plotted in Fig. 6. The comparison is carried out at different values of $n_{H}$ and $n_{M}$. Clearly, the maximum achievable efficiency for the $n$ pulse 16-PPM scheme is about $85 \%$ and it occurs at $n_{M}=8$ pulses, whereas for the hybrid $n$-BPSK 16-PPM scheme, the bandwidth-utilization efficiency could approach levels higher than $100 \%$. Specifically at $n_{H}=11$ pulses, this efficiency has a maximum of $144.33 \%$. After reaching this maximum value, the efficiency decreases again by increasing the value of $n_{H}$ until approaching a value of $100 \%$ at $n_{H}=16$. This latter case represents the ordinary BPSK transmission. Thus, the proposed modulation scheme is strongly intended to be used for applications that require high bandwidth utilization.

\section{TRAnsmitter And Receiver Block Diagram AND DECODING ALGORITHM}

The bit encoding for hybrid BPSK-modified MPPM scheme is generally carried by the same encoding techniques of ordinary MPPM scheme. However, there is no known efficient method of encoding ordinary MPPM symbols [14]. The simplest, but inefficient, encoding scheme for ordinary MPPM is to encode each symbol by the integer number of bits resulting from taking the logarithm of number of available symbols in the system. As an example of such encoding when implemented for hybrid 4-BPSK 9-PPM scheme, each hybrid frame is encoded only by 10 bits and the remaining fraction of bits $(0.97 \mathrm{bit})$ is discarded. Clearly, in this case not all the available symbols are used for transmission and there is an optimal bit-symbol mapping that results in the lowest BER. Another simple and more efficient encoding scheme for ordinary MPPM is to create compound symbols by cascading enough MPPM symbols to ensure that the total number just exceeds a power of 2 and then encoding suitably larger blocks of information bits into this compound 


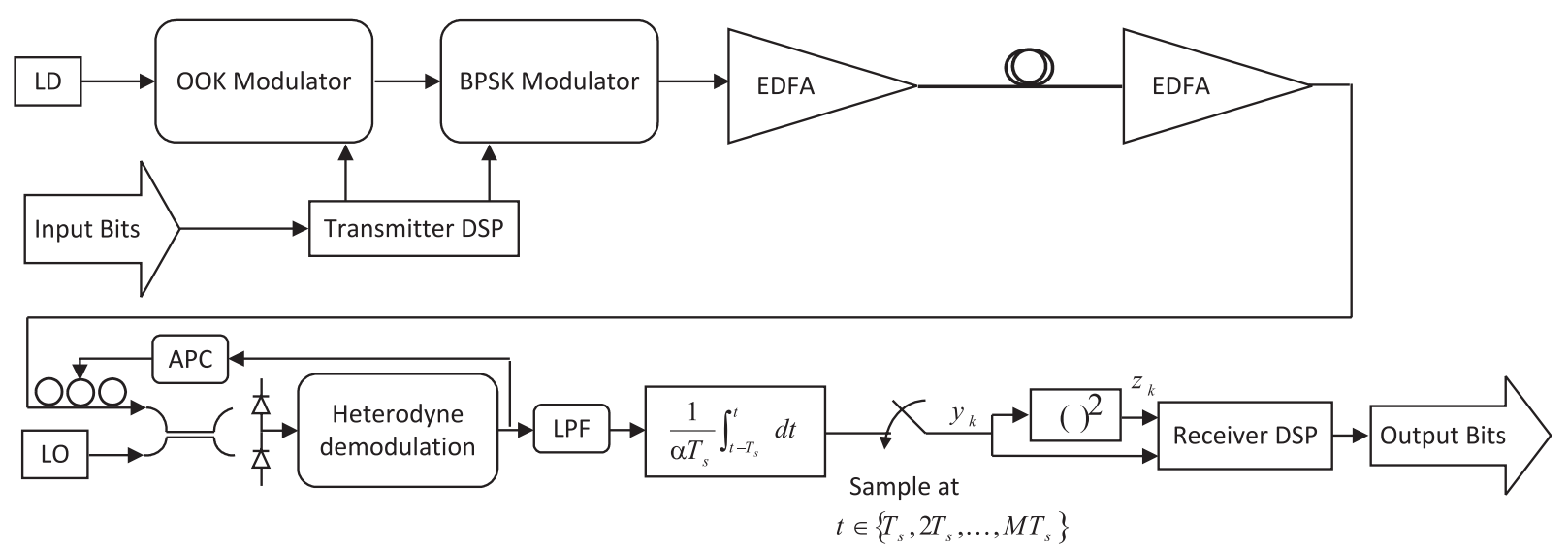

Fig. 7. Transmitter and receiver block diagram for hybrid BPSK-modified MPPM scheme. The parameter $\alpha=2 \mathcal{R} A_{L}$ for a homodyne receiver or $\alpha=\mathcal{R} A_{L}$ for a heterodyne receiver.

signal set [15]. Although the bit encoding and decoding for MPPM and consequently for hybrid BPSK-modified MPPM are not in the scope of this paper, they may represent important design issues that could be considered in future research works.

The transmitter and receiver block diagram for hybrid BPSKmodified MPPM systems are shown in Fig. 7. At the transmitter side, the transmitted data bits are first fed to a digital signal processing (DSP) device, which divides them into several blocks. Each block contains $\log _{2}\left(\begin{array}{c}M \\ n_{H}\end{array}\right)+n_{H}$ bits. The first $\log _{2}\left(\begin{array}{c}M \\ n_{H}\end{array}\right)$ bits determine the transmitted MPPM symbol, i.e., the positions of the $n_{H}$ signal pulses within the MPPM frame, while, the remaining $n_{H}$ bits are used to modulate these signal pulses with ordinary BPSK modulation. That is, the first $\log _{2}\left(\begin{array}{c}M \\ n_{H}\end{array}\right)$ bits of the data block are encoded in the hybrid frame by intensity modulating a continuous wave $(\mathrm{CW})$ laser source, generating $n_{H}$ optical pulses. The remaining $n_{H}$ bits are encoded in the hybrid frame by phase modulating the $n_{H}$ optical pulses in the hybrid frame.

At the receiver side, coherent detection, for both BPSK symbols and MPPM frames, is adopted to increase the receiver sensitivity. In addition, the coherent receiver implements balanced optical detectors in order to minimize the noise generated from local oscillator (LO). Generally, for coherent receivers limited by optical amplifier noise, both heterodyne and homodyne detection receivers achieve same performance [1]. The photodetector output of the homodyne receiver (or the IF demodulator output of the heterodyne receiver) is then fed to a slot integrator. The slot integrator output is then squared and both the integrator output and its square value are passed to a DSP to decode the received hybrid frame. The DSP selects the $n_{H}$ pulses with the maximum square values and decode them as the signaling pulses in the received frame. This is the same as normal decoding of MPPM schemes [13]. After decoding the first $\log _{2}\left(\begin{array}{c}M \\ n_{H}\end{array}\right)$ bits of the transmitted data block, the DSP proceeds to decode the remaining $n_{H}$ bits by comparing the slot integrator values for the predetermined $n_{H}$ signal slots with zero. Obviously, the slot is decoded as one if its integrator output is larger than zero; otherwise, it is decoded as zero.

\section{Decoding Algorithm}

1) Perform coherent detection for received hybrid frame by homodyne or heterodyne demodulation.

2) Get received signal amplitude in each slot.

3) Determine the energy received in each slot by integrating the received signal amplitudes and squaring the integrator output samples.

4) Determine $n_{H}$ signal slots of received hybrid frame by selecting the $n_{H}$ slots with the highest energies.

5) Decode the BPSK signals of these slots by comparing their integrated samples to zero.

\section{ENHANCING BPSK USING HYBRID BPSK-MODIFIED MPPM SCHEME}

One major goal of proposing hybrid BPSK-modified MPPM scheme is to enhance the performance of ordinary BPSK modulation in optical communications. Clearly, instead of transmitting a consecutive stream of BPSK pulses, each with a relatively small power, we transmit less number of high power BPSK pulses and exploit the positions of these pulses to transmit more bits in the hybrid frame. The enhancement achieved in the performance of BPSK using the hybrid scheme is further investigated in the following sections.

\section{A. Performance Evaluation}

In this section, we aim at evaluating the bit-error rate (BER) of the proposed hybrid BPSK-modified MPPM. In this evaluation, the optical fiber is considered as the transmission medium for the proposed scheme. Also, we assume that the optical fiber span contains many optical amplifiers so that the system is limited by the amplified spontaneous emission (ASE) noise generated from these amplifiers [1]. Generally, the extension of the carried analysis for both free space and deep space optical communications is straightforward and can be achieved using appropriate optical propagation models for these channels.

Here, the input data stream is divided into continuous blocks, each block contains $\log _{2}\left(\begin{array}{c}M \\ n_{H}\end{array}\right)+n_{H}$ bits. Each data block is encoded to form one hybrid frame. Thus, the bit-error rate of 
the hybrid BPSK-modified MPPM scheme can be calculated as the average BER of two groups of bits as follows:

$$
\begin{aligned}
\mathrm{BER}_{H}= & \frac{\log _{2}\left(\begin{array}{c}
M \\
n_{H}
\end{array}\right)}{n_{H}+\log _{2}\left(\begin{array}{c}
M \\
n_{H}
\end{array}\right)} \times \mathrm{BER}_{\mathrm{MPPM}}+\frac{n_{H}}{n_{H}+\log _{2}\left(\begin{array}{c}
M \\
n_{H}
\end{array}\right)} \\
& \left\{\left(1-\mathrm{SER}_{\mathrm{MPPM}}\right) \times \mathrm{BER}_{\mathrm{BPSK}}+\frac{\mathrm{SER}_{\mathrm{MPPM}}}{n_{H}\left[\left(\begin{array}{c}
M \\
n_{H}
\end{array}\right)-1\right]}\right. \\
& \left.\sum_{i=1}^{\left\lfloor n_{H}, M-n_{H}\right\rfloor} X_{i}\left[\left(n_{H}-i\right) \times \mathrm{BER}_{\mathrm{BPSK}}+\frac{i}{2}\right]\right\}
\end{aligned}
$$

where $\mathrm{BER}_{\mathrm{BPSK}}$ is the bit error rate of ordinary BPSK,

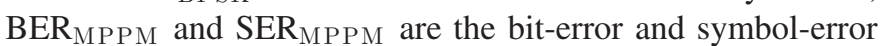
rates for ordinary MPPM scheme, respectively. Generally, it is difficult to get a closed form to calculate BER MPPM $_{\text {. However, }}$ for MPPM scheme that transmits $L$ bits per frame, where $L$ is an integer number, the relation between $\mathrm{SER}_{\mathrm{MPPM}}$ and $\mathrm{BER}_{\mathrm{MPPM}}$ is upper bounded by [16]

$$
\mathrm{BER}_{\mathrm{MPPM}} \leqslant \frac{2^{L-1}}{2^{L}-1} \times \mathrm{SER}_{\mathrm{MPPM}}
$$

The simplest way to map the encoded bits into MPPM symbols is to use a number of symbols equals to $2^{L}$, i.e.. an integer power of two. In this case, the number of neglecting symbols in the MPPM system is $\left(\begin{array}{c}M \\ n_{H}\end{array}\right)-2^{L}$. Clearly, the bound of (6) is approached if the number of neglected symbols is small compared to the used ones.

Here, $X_{i}$ represents number of MPPM symbols that differ with $i$ slots from the transmitted symbol. The value of $X_{i}$ can be calculated as

$$
X_{i}=\left(\begin{array}{c}
n_{H} \\
i
\end{array}\right)\left(\begin{array}{c}
M-n_{H} \\
i
\end{array}\right) .
$$

Clearly, we have $\sum_{i=1}^{\left\lfloor n_{H}, M-n_{H}\right\rfloor} X_{i}=\left(\begin{array}{c}M \\ n_{H}\end{array}\right)-1$ which represent all the available symbols except the transmitted one. The first term in (5) accounts for the bit-error rate that occurs to the group of $\log _{2}\left(\begin{array}{c}M \\ n_{H}\end{array}\right)$ bits which is the same as the bit error rate of ordinary MPPM. The second term of the equation accounts for bit-error rate of the remaining $n_{H}$ bits. Clearly, this term consists of two parts; the first part considers the case when $n_{H}$ signal slots are correctly decoded, while the second part considers the case when they are wrongly decoded. In the first part, the bit-error rate for the transmitted $n_{H}$ bits is the same as

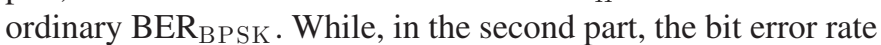
is an average between $\mathrm{BER}_{\mathrm{BPSK}}$ and 0.5 (random decoding) based on the average number of wrongly decoded slots.

Clearly, due to the phase demodulation, coherent detection is used instead of direct detection in demodulating the proposed hybrid scheme. However, in the analysis of pre-amplifiered coherent receivers there are many types of noise sources. The main three noise sources are the ASE noise, the local oscillator noise, and the detector noise, which includes both the shot and thermal noises. Here, we use a dual-photodiode balanced receiver to reduce the LO noise and increase the signal power. Also, we assume that the detector shot noise variance is much larger than that of the thermal noise so that the detector thermal noise can be neglected.

To simplify the receiver structure and for optimal signal mixing, the polarization of the received signal is aligned to that of the LO laser using automatic polarization control APC as indicated in Fig. 7. The polarization control algorithm should be able to provide endless polarization control without reset. Many methods can control the polarization though APC [17]. However, the coherent detection could also be realized using polarization-diversity receiver which may be considered in future research work [1]. In this receiver which consists of two balanced detectors, the coherent detection is independent on the polarization state of the received signal.

Here, we recall the general model for coherent detection of optical phase modulated signals to develop the coherent detection model for the proposed hybrid scheme [1]. The electric field equations for both the received optical field and the LO field are given by

$$
\begin{aligned}
E_{r}(t) & =\left[A_{s}(t) e^{j \phi_{s}(t)}+n_{x}(t)\right] e^{j \omega_{c} t} \hat{x}+n_{y}(t) e^{j \omega_{c} t} \hat{y} \\
E_{L O}(t) & =\left[A_{L}+n_{L}(t)\right] e^{j \omega_{L O} t} \hat{x}
\end{aligned}
$$

where $A_{s}(t), \phi_{s}(t)$, and $\omega_{c}$ are the amplitude, phase, and frequency of the received signal, respectively, $\hat{x}$ is the polarization of the signal, $\hat{y}$ is the polarization orthogonal to $\hat{x}, n_{x}(t)$ and $n_{y}(t)$ are the ASE noise in the polarizations of $\hat{x}$ and $\hat{y}$, respectively, $A_{L}$ and $\omega_{L O}$ are the amplitude and frequency of the local oscillator, respectively, and $n_{L}(t)$ is the local oscillator optical noise, originated from the relative intensity noise (RIN) of local oscillator laser. The amplifier noise can be expressed as a complex representation:

$$
n_{x}(t)=n_{x 1}(t)+j n_{x 2}(t)
$$

where $n_{x 1}(t)$ and $n_{x 2}(t)$ are Gaussian distributed with zero mean and variance $\sigma_{x}^{2}=S_{n_{s}} B_{o} / 2 . S_{n_{s}}$ is the power spectral density of the received spontaneous emission (ASE noise) in each polarization $\left(\hat{x}\right.$ or $\hat{y}$ ) and $B_{o}$ is the optical bandwidth of the received optical filter [1]. Notice that $\operatorname{var}\left\{n_{x}(t)\right\}=2 \sigma_{x}^{2}=$ $S_{n_{s}} B_{o}$. In our analysis below, we ignore the thermal noise for simplicity. This would give more insight into the problem under consideration. However, as an additive noise, the effect of thermal noise can be added to the signal afterwards.

The electric field at the input of the upper photodiode of the dual balanced detector (at the upper output of the optical coupler) is $\left[E_{r}(t)+E_{L O}(t)\right] / \sqrt{2}$ and the photo-current is equal to

$$
i_{1}(t)=\frac{\mathcal{R}}{2}\left|E_{r}(t)+E_{L O}(t)\right|^{2}+i_{s h 1}
$$

where $\mathcal{R}$ is the photodiode responsively and $i_{s h 1}$ is shot noise associated with upper photodiode. The electric field at the input of the lower photodiode (at the lower output of the optical coupler) is $\left[E_{r}(t)-E_{L O}(t)\right] / \sqrt{2}$ and the photo-current is equal to

$$
i_{2}(t)=\frac{\mathcal{R}}{2}\left|E_{r}(t)-E_{L O}(t)\right|^{2}+i_{s h 2}
$$


where $i_{s h 2}$ is the shot noise associated with the lower photodiode. The overall output current of the balanced detector is given as

$$
i(t)=i_{1}(t)-i_{2}(t)=2 \mathcal{R} \Re\left\{E_{r}(t) \times E_{L O}^{*}(t)\right\}+i_{s h}
$$

where $i_{s h}=i_{s h 1}-i_{s h 2}$ is the overall shot noise current of the two photodiodes. Neglecting the small effect of beating of ASE noise with LO noise, the output current is given by

$$
\begin{aligned}
i(t)=2 \mathcal{R} & A_{L} A_{s}(t) \cos \left[\omega_{I F} t+\phi_{s}(t)\right]+2 \mathcal{R} \Re\left\{\left[A_{L} n_{x}(t)\right.\right. \\
+ & \left.\left.A_{s}(t) e^{j \phi_{s}(t)} n_{L}(t)\right] e^{j \omega_{I F} t}\right\}+i_{s h}
\end{aligned}
$$

where $\omega_{I F}=\omega_{c}-\omega_{L O}$ is the intermediate frequency. Generally, the value of signal-LO beating noise is small compared to that of both LO-ASE beating noise and detector shot noise. Also, the noise generated from local oscillator $n_{L}(t)$ is totally eliminated using the dual balanced receiver. Furthermore, in the usual case where the LO power is significantly larger than the received power, the dominant noise source is LO-ASE beating. In this case, we get an amplifier-noise limited system and the balanced detector current can be simplified as

$$
\begin{array}{rl}
i(t)= & 2 \mathcal{R} A_{L} A_{s}(t) \cos \left[\omega_{I F} t+\phi_{s}(t)\right] \\
& +2 \mathcal{R} \Re\left\{A_{L} n_{x}(t) e^{j \omega_{I F} t}\right\} \\
=2 & \mathcal{R} A_{L} A_{s}(t) \cos \left[\omega_{I F} t+\phi_{s}(t)\right] \\
& +2 \mathcal{R} A_{L} n_{x 1}(t) \cos \omega_{I F} t-2 \mathcal{R} A_{L} n_{x 2}(t) \sin \omega_{I F} t
\end{array}
$$

In a heterodyne receiver, this current would pass to a subcarrier synchronous detector (to remove the effect of the IF frequency), followed by a low-pass filter (LPF) with electrical bandwidth of $B_{e}=1 / 2 T_{s}$, where $T_{s}$ is the pulse duration. The LPF output can be expressed as

$$
i_{L P F}(t)=\mathcal{R} A_{L} A_{s}(t) \cos \left[\phi_{s}(t)\right]+\mathcal{R} A_{L} n(t)
$$

where, $n(t)$ is a band-limited noise of bandwidth $B_{e}$. Also, it should be noticed that during any time slot within a frame, $A_{s}(t) \in\left\{0, A_{H}\right\}$, for some constant $A_{H}>0$, and $\phi_{s}(t) \in\{0, \pi\}$. That is, $A_{s}(t) \cos \left[\phi_{s}(t)\right] \in\left\{-A_{H}, 0, A_{H}\right\}$. Next $i_{L P F}(t)$ is passed through an integrator over a slot duration. The integrator output over slot $k \in\{1,2, \ldots, M\}$, also called the decision random variable, is

$$
\begin{aligned}
y_{k} & \stackrel{\text { def }}{=} \frac{1}{\mathcal{R} A_{L} T_{s}} \int_{(k-1) T_{s}}^{k T_{s}} i_{L P F}(t) d t= \\
& =\left\{\begin{array}{lr}
A_{H}+n_{k} ; & \text { if a pulse was transmitted, modulated by } 1 \\
n_{k} ; & \text { if a pulse was not transmitted } \\
-A_{H}+n_{k} ; & \text { if a pulse was transmitted, modulated by } 0
\end{array}\right.
\end{aligned}
$$

where $T_{s}$ is the slot duration and

$$
n_{k} \stackrel{\text { def }}{=} \frac{1}{T_{s}} \int_{(k-1) T_{s}}^{k T_{s}} n(t) d t .
$$

It is clear that, for any $k \in\{1,2, \ldots, M\}, n_{k}$ is a Gaussian random variable with mean and variance given by

$$
\begin{aligned}
& \mu_{n} \stackrel{\text { def }}{=} E\left\{n_{k}\right\}=0 \\
& \sigma_{n}^{2} \stackrel{\text { def }}{=} \operatorname{var}\left\{n_{k}\right\}=\frac{1}{2} S_{n_{s}} B_{e}=\frac{S_{n_{s}}}{4 T_{s}},
\end{aligned}
$$

respectively.

In a homodyne receiver, we can get the same decision random variable as given in (16) if we used an integrator $\frac{1}{2 \mathcal{R} A_{L} T_{s}} \int_{(k-1) T_{s}}^{k T_{s}}(\cdot)$ rather. For either a heterodyne or homodyne receiver, the output of slot integrator $y_{k}$ is one of three Gaussian random variables, which are $A_{H}+n_{k}, n_{k}$, or $-A_{H}+n_{k}$, associated with the transmission of an optical pulse modulated by one, transmission of no optical pulse, or transmission of an optical pulse modulated by zero, respectively. The probability distributions of $y_{k}$ for these three cases are given by

$$
\begin{gathered}
p_{1}\left(y_{k}\right) \stackrel{\text { def }}{=} p\left(y_{k} \mid 1\right)=\frac{1}{\sigma_{n} \sqrt{2 \pi}} e^{-\left(y_{k}-A_{H}\right)^{2} / 2 \sigma_{n}^{2}} \\
p_{0}\left(y_{k}\right) \stackrel{\text { def }}{=} p\left(y_{k} \mid 0\right)=\frac{1}{\sigma_{n} \sqrt{2 \pi}} e^{-y_{k}^{2} / 2 \sigma_{n}^{2}} \\
p_{-1}\left(y_{k}\right) \stackrel{\text { def }}{=} p\left(y_{k} \mid-1\right)=\frac{1}{\sigma_{n} \sqrt{2 \pi}} e^{-\left(y_{k}+A_{H}\right)^{2} / 2 \sigma_{n}^{2}},
\end{gathered}
$$

respectively.

The receiver gets the $n_{H}$ slots with the highest energy in the received hybrid frame by squaring the integrator output. The resultant random variable $z_{k}=y_{k}^{2}$ takes one of two cases: $\left(A_{H} \pm n_{k}\right)^{2}$ or $n_{k}^{2}$, depending on the transmission of an optical pulse or no optical pulse, respectively. The probability distribution of $z_{k}$ in the case of transmitting an optical pulse is a non-central chi-square $\chi^{2}$ with one degree of freedom, given by

$$
\begin{aligned}
p_{1}\left(z_{k}\right) \stackrel{\text { def }}{=} p\left(z_{k} \mid 1\right)= & \frac{1}{2 \sigma_{n}^{2}} e^{-\left(z_{k}+A_{H}^{2}\right) / 2 \sigma_{n}^{2}}\left(\frac{A_{H}^{2}}{z_{k}}\right)^{\frac{1}{4}} \\
& \times I_{-\frac{1}{2}}\left(\frac{A_{H}}{\sigma_{n}^{2}} \sqrt{z_{k}}\right), \quad z_{k}>0
\end{aligned}
$$

where $I_{\alpha}(\nu)$ is the $\alpha$ th-order modified Bessel function of the first kind, while, the probability distribution of $z_{k}$ in the case of no optical pulse transmission, is a central chi-square with one degree of freedom (also called gamma distribution), given by

$$
p_{0}\left(z_{k}\right) \stackrel{\text { def }}{=} p\left(z_{k} \mid 0\right)=\frac{1}{\sigma_{n} \sqrt{2 \pi z_{k}}} e^{-z_{k} / 2 \sigma_{n}^{2}}, \quad z_{k}>0 .
$$

Clearly, the receiver selects the $n_{H}$ slots with the highest values of $z_{k}$ (highest energies) and decodes them as the transmitted signal pulses. The probability of error in such a decoding is the same as the symbol-error rate of the ordinary MPPM scheme, which is given by [13], with slight modifications. We proceed as follows. The probability of correct symbol detection equals to the probability that $n_{H}$ signal slot energies are all greater than the largest energy of $M-n_{H}$ non-signal slot. Let $Z$ be the largest non-signal slot energy, then the probability of errors 
in such symbol decoding is given by

$$
\begin{aligned}
\operatorname{SER}_{M P P M}=1 & -\int_{0}^{\infty}\left(M-n_{H}\right) P_{0}(Z)^{M-n_{H}-1} \\
& \times p_{0}(Z)\left[1-P_{1}(Z)\right]^{n_{H}} d Z,
\end{aligned}
$$

where $p_{1}(Z)$ and $p_{0}(Z)$ are the probability density functions (pdfs) of $Z$ in signal and non-signal slots, and are given by (20) and (21), respectively. Also, $P_{1}(Z)$ and $P_{0}(Z)$ denote its cumulative distributions for both signal and non-signal slots, respectively. Clearly, the equation consider all probabilities for $M-n_{H}$ non-signal slots to have energy less than the $n_{H}$ signal slots.

After getting the positions of the $n_{H}$ signal slots in the received hybrid frame, the receiver proceeds to decode these BPSK modulated signal slots to find the remaining $n_{H}$ bits of the transmitted data block. Clearly, the decoding decision will be based on the values of slot integrator for the predetermined signal slots. The integrator output of these slots takes one of two values which are $A_{H}+n_{k}$ or $-A_{H}+n_{k}$, depending on the transmission of data bit one or zero on the optical pulse, respectively. With the optimal threshold set at zero, the error probability in decoding these BPSK symbols (or bits) is the same as the bit error rate of ordinary BPSK [18]:

$$
\begin{aligned}
\operatorname{BER}_{\mathrm{BPSK}} & =\frac{1}{2} \int_{-\infty}^{0} p\left(y_{k} \mid 1\right) d y+\frac{1}{2} \int_{0}^{\infty} p\left(y_{k} \mid-1\right) d y \\
& =\frac{1}{2} \operatorname{erfc}\left(\frac{A_{H}}{\sigma_{n} \sqrt{2}}\right) .
\end{aligned}
$$

Substituting the results of (6), (22), and (23) into (5), we obtain an upper bound on $\mathrm{BER}_{H}$ for hybrid BPSK-modified MPPM scheme.

\section{B. Numerical Results}

In this section, we compare the performance achieved by the proposed hybrid BPSK-modified MPPM with that achieved by ordinary BPSK. For fair performance comparison, we assume the usage of same frame size and same transmission rate for both schemes. Clearly, with the same transmission bit rate the comparison is carried in terms of the achievable bit-error rate. Although the comparison is carried for optical fiber channels, it can be extended easily for other optical channels by considering the appropriate optical transmission models. Specifically, the evaluations are performed for two hybrid modulation schemes which are hybrid 3-BPSK 9-PPM and hybrid 6-BPSK 22-PPM. For these schemes, the numbers of transmitted bits per frame are 9.1 bits and 22.4 bits, respectively, which are slightly larger than that of the ordinary BPSK schemes ( 9 and 22 bits, respectively). Clearly, these selections achieve near the same transmission rates for both hybrid and ordinary schemes with larger rates for hybrid ones. However, if numbers of transmitted optical pulses per hybrid frames are increased above the mentioned values, the hybrid schemes can achieve higher bandwidth-utilization efficiencies than that of ordinary BPSK ones.

The noise considered in the evaluations is assumed to be dominated by optical amplifier ASE noise. This noise is mod-

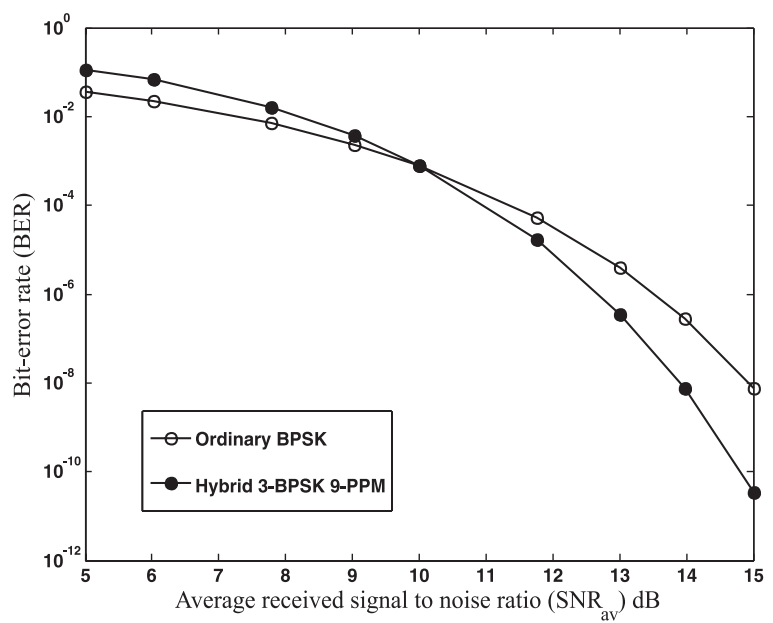

Fig. 8. Bit-error rate versus average received signal-to-noise ratio for hybrid 3-BPSK 9-PPM scheme.

eled as a Gaussian noise with zero mean and variance of $\sigma_{n}^{2}$. Also, for received signal power, the comparisons are performed under an average power constraint, which is imposed in many optical communications systems. For optical fiber based systems, this constraint rises in the case of long haul transmission where optical repeaters with limited average power is used and also in the case of DWDM transmission, where thousands of optical carriers are launched together in a single core and the total average power launch to the fiber must not exceed a maximum value to avoid fiber fusing [19]. For non-fiber systems, the average power constraint is imposed when the transmitter has limited power resources. This occurs in FSO systems working with solar energy and space optical communications between spacecrafts. Clearly, the modulation scheme that achieves higher performance at the same average power level is highly desired in these cases.

The average power for hybrid BPSK-modified MPPM and ordinary BPSK schemes can be expressed as $P_{\mathrm{av}_{H}}=A_{H}^{2} \cdot n_{H} / M$ and $P_{\mathrm{av}_{B}}=A_{B}^{2}$, respectively, where $A_{H}$ and $A_{B}$ are the amplitudes of demodulated signals for hybrid BPSK-modified MPPM and ordinary BPSK schemes, respectively. Clearly, under the average power constraint, we have $P_{\mathrm{av}_{H}}=P_{\mathrm{av}_{B}}$ and $A_{H}^{2}=A_{B}^{2} \cdot M / n_{H}$. Moreover, with the same transmission rates for both hybrid and ordinary schemes, the average power constraint implies the transmission of the same energy per bit. In this case, the average received signal to noise ratio for both schemes is defined as

$$
\mathrm{SNR}_{\mathrm{av}}=\frac{P_{\mathrm{av}}}{\sigma_{n}^{2}}=\frac{A_{H}^{2} \cdot n_{H}}{M \sigma_{n}^{2}}=\frac{A_{B}^{2}}{\sigma_{n}^{2}}
$$

Figs. 8 and 9 show the first performance comparison between hybrid and ordinary schemes under average received power constraint. The comparison is carried in terms of achieved bit-error rate versus average signal to noise ratio. The results for hybrid 3-BPSK 9-PPM scheme are shown in Fig. 8. Clearly, for small values of $\mathrm{SNR}_{\mathrm{av}}$ (less than $10 \mathrm{~dB}$ ), ordinary BPSK achieves better performance (less BER) than that of hybrid scheme. Specifically, at $\mathrm{SNR}_{\mathrm{av}}=5 \mathrm{~dB}$ (very small $\mathrm{SNR}_{\mathrm{av}}$ ), the BER of 


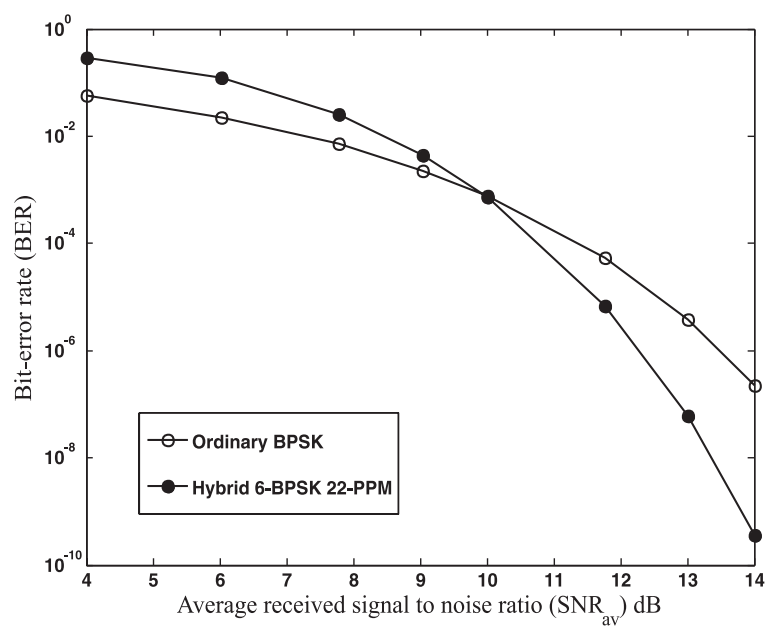

Fig. 9. Bit-error rate versus average received signal-to-noise ratio for hybrid 6-BPSK 22-PPM scheme.

ordinary BPSK is 0.0368 which is about three times less than that of hybrid 3-BPSK 9-MPPM scheme. However, increasing $\mathrm{SNR}_{\mathrm{av}}$ level beyond $10 \mathrm{~dB}$ (critical value) results in a better performance for hybrid scheme. Numerically, at $\mathrm{SNR}_{\mathrm{av}}=13 \mathrm{~dB}$, the BER achieved by hybrid scheme is one order of magnitude smaller than that of ordinary BPSK. In other words, at $\mathrm{BER}=10^{-7}$, a reduction of $1 \mathrm{~dB}$ in $\mathrm{SNR}_{\mathrm{av}}$ could be achieved by the hybrid scheme. Also, the BER gap between the two schemes is much increased by increasing the $\mathrm{SNR}_{\mathrm{av}}$ level.

However, these results can be interpreted as: at small levels of $\mathrm{SNR}_{\mathrm{av}}$, the probability of correct detection of MPPM symbols is small because it depends on the relative difference between received signal and noise energies. This is in contrast to the probability of correct detection of BPSK symbols which depends on the sign of received signal amplitude, not its energy, which results in much higher noise margin than that of energy detection for MPPM scheme. However, increasing $\mathrm{SNR}_{\mathrm{av}}$ levels results in much better energy detection for MPPM scheme than sign detection of ordinary BPSK scheme.

It is worth noticing that the range of practical interest of BER (less than $10^{-4}$ ) lies inside the range where the hybrid scheme performs better. This makes the hybrid scheme is the better choice. Although forward error correction (FEC) schemes are commonly used with raw BER (without FEC) higher than 1e3 , but the price is the significant increase in the transmission overheads associated with these schemes. Clearly, at high average transmission optical power, the proposed hybrid modulation format could achieve the required BER without implementing FEC which in turn maintains the transmission efficiency of the optical fiber communication system.

Generally, as indicated in Fig. 9, increasing the frame size results in a better BER performance for hybrid BPSK-modified MPPM scheme. The figure shows the BER achieved by hybrid 6-BPSK 22-PPM scheme. Again, this hybrid frame selection achieves near the same bandwidth-utilization for both hybrid and ordinary schemes with an advantage to the hybrid one. Clearly, at all $\mathrm{SNR}_{\mathrm{av}}$ levels, hybrid 6-BPSK 22-PPM scheme achieves less BER than that of hybrid 3-BPSK 9-PPM scheme.
Numerically, at $\mathrm{SNR}_{\mathrm{av}}$ equals $13 \mathrm{~dB}$, the BER for hybrid 6BPSK 22-PPM is six times lower than that achieved by hybrid 3-BPSK 9-PPM scheme. Also, the performance gap between the two hybrid schemes increases by increasing the $\mathrm{SNR}_{\mathrm{av}}$ level. Moreover, the critical $\mathrm{SNR}_{\mathrm{av}}$ at which both hybrid and ordinary schemes achieve the same BER is reduced for larger hybrid frame sizes.

However, this enhancement comes from the significant increase in the transmitted pulse power for schemes with longer frames. Here, the peak power in hybrid 6-BPSK 22-PPM frame is about $122 \%$ higher than that of hybrid 3-BPSK 9-PPM frame with the same average transmitted power.

The asymptotic power efficiency of the proposed hybrid modulation scheme could be computed as [20]

$$
\gamma=\frac{d_{\min }^{2}}{4 \varepsilon_{b}}=\frac{\frac{A_{H}^{2} \times T}{M}}{\frac{A_{H}^{2} \times T \times 2 n}{M \times\left(n+\log _{2}\left(\begin{array}{c}
M \\
n
\end{array}\right)\right)}}=\frac{n+\log _{2}\left(\begin{array}{c}
M \\
n
\end{array}\right)}{2 n}
$$

where, $\gamma$ is the asymptotic power efficiency, $d_{\min }$ is the minimum distance between any two symbols of the hybrid scheme and $\varepsilon_{b}$ is the energy per bit. The asymptotic power efficiencies for hybrid 3-BPSK 9-PPM and hybrid 6-BPSK 22-PPM schemes are 1.5634 and 1.8489 , respectively which are better than the ordinary BPSK case $(\gamma=1)$. Clearly, these values indicate that the energy is efficiently allocated in the hybrid scheme to achieve better separation distance between the hybrid symbols.

It should be mentioned that the performance enhancement achieved by hybrid schemes comes with price of increasing peak power levels. Also, enhancing system performance by increasing hybrid frame size comes with additional increase in peak power levels. Obviously, these high levels of peak power raise the amount of nonlinear distortion and cross phase noise, which is not desirable for high speed optical fiber communications. Moreover, delivering these high power levels could be too difficult due to saturation of optical amplifiers. Practically, this increase in peak power may limit the performance of hybrid scheme to values lower than that of ordinary schemes when the same maximum peak power level is allowed.

\section{ENHANCING MPPM USING HYBRID BPSK-MODIFIED MPPM SCHEME}

Another goal of proposing hybrid BPSK-modified MPPM scheme is to enhance the performance of ordinary MPPM scheme in optical communications systems. As mentioned earlier, one of the main enhancements of this hybrid scheme is the significant increase in bandwidth utilization efficiency. Indeed, we can either increase number of transmitted bits per frame while using same number of transmitted optical pulses, or inversely, we can transmit same number of bits per frame while using less number of optical pulses. Under an average power constraint, the latter results in increasing the energy per pulse, which in turn results in a better detection and less symbol-error rates. The enhancement achieved in the performance of MPPM 
using the hybrid scheme is further investigated in the following sections.

\section{A. Performance Evaluation}

In this section, we aim at evaluating the symbol-error rate (SER) for the proposed hybrid BPSK-modified MPPM and compare it to the SER of ordinary MPPM. In our evaluation we consider the optical fiber as the transmission medium for the hybrid scheme. Also, we assume that the system is limited by the ASE noise generated from successive optical amplifiers in the fiber span. Here, the input data stream is divided into continuous blocks, each block contains $\log _{2}\left(\begin{array}{c}M \\ n_{H}\end{array}\right)+n_{H}$ bits. Each data block is encoded to form one hybrid frame. Thus, the SER for the hybrid BPSK-modified MPPM scheme is the result of two independent events and is given by

$$
\mathrm{SER}_{H}=1-\left(1-\mathrm{SER}_{\mathrm{MPPM}}\right) \times\left(1-\mathrm{BER}_{\mathrm{BPSK}}\right)^{n_{H}}
$$

where $\mathrm{BER}_{\mathrm{BPSK}}$ and $\mathrm{SER}_{\mathrm{MPPM}}$ are the bit-error rate of ordinary BPSK and the symbol-error rate of ordinary MPPM, respectively. The first parentheses in (26) accounts for the event that the MPPM symbol is correctly decoded and its pulses' positions are correctly determined. The second parentheses of the equation accounts for the event that all BPSK symbols within the hybrid frame are correctly decoded. Clearly, the hybrid frame is considered to be correctly decoded only when both pulses' positions and BPSK symbols are correctly identified.

Again, in the carried analysis, we implement dual-photodiode balanced receiver to reduce the $\mathrm{LO}$ noise, so that the dominant noise source is the ASE noise. Also, we assume that the detector shot noise variance is much larger than that of the thermal noise so that the detector thermal noise can be neglected. The electric field for both the received optical field and the LO field are given by (8). The expressions for both SER MPPM $_{\text {and }}$ BER $_{\text {BPSK }}$ are still given by (22) and (23), respectively. Substituting into (26), we obtain the exact $\mathrm{SER}_{H}$ for the proposed hybrid BPSKmodified MPPM scheme.

For the purpose of performance comparison, we recall the SER expression for coherent detection of MPPM scheme. Clearly, the SER is still given by equation (22), but with different distributions, integration limits, and different number of optical pulses per frame $n_{M}$.

However, using a similar procedure as described in [21], the probability distributions of slot energy for coherent detected MPPM can be obtained as

$$
\begin{aligned}
& p_{1}\left(z_{k}\right)=\frac{1}{\sigma_{n} \sqrt{2 \pi}} e^{-\left(z_{k}-A_{M}\right)^{2} / 2 \sigma_{n}^{2}} \\
& p_{0}\left(z_{k}\right)=\frac{1}{\sigma_{n} \sqrt{2 \pi}} e^{-z_{k}^{2} / 2 \sigma_{n}^{2}} .
\end{aligned}
$$

\section{B. Numerical Results}

In this section, we compare the performance achieved by the proposed hybrid BPSK-modified MPPM scheme with that achieved by ordinary MPPM scheme. Due to the difficulty in computing the bit-error rate of ordinary MPPM, the comparison is carried out in terms of the achievable symbol-error rate. For fair performance comparison, we assume the usage of the same frame size and the same transmission rate for both schemes. Specifically, the evaluations are performed at two different frame sizes, namely, $M=12$ and $M=24$ slots. For the case of $M=12$, two comparisons are carried out. Firstly, 3 -pulse 12-PPM is compared to hybrid 2-BPSK 12-PPM and secondly, 6-pulse 12-PPM is compared to hybrid 3-BPSK 12PPM. For these hybrid schemes, the numbers of transmitted bits per frame are 8.04 bits and 10.78 bits, respectively, which are slightly larger than that of the ordinary MPPM schemes (7.78 bits and 9.85 bits, respectively). Clearly, these selections achieve nearly the same transmission rates for both hybrid and ordinary schemes with larger rates for the hybrid ones. For the case $M=24$, two comparisons are also performed. Particularly, we compare 6-pulse 24-PPM scheme to hybrid 4-BPSK 24-PPM scheme and compare 12-pulse 24-PPM scheme to hybrid 6-BPSK 24-PPM scheme. Again, in these comparisons, the hybrid schemes achieve higher transmission rates than that of ordinary MPPM schemes, which represents an extra advantage in favor of selected hybrid schemes.

Generally, coherent detection results in a better receiver sensitivity than direct detection, but it requires more complex receiver structure. we compare the performance achieved by the proposed coherent detection hybrid BPSK-modified MPPM scheme to that achieved by both coherent detection and direct detection MPPM schemes. Also, in all these cases, the system noise is assumed to be dominated by optical amplifier ASE noise.

Furthermore, our comparisons are performed under the average power constraint. The average power for hybrid BPSKmodified MPPM and ordinary MPPM schemes can be expressed as $P_{\mathrm{av} H}=A_{H}^{2} \cdot n_{H} / M$ and $P_{\mathrm{av} M}=A_{M}^{2} \cdot n_{M} / M$, respectively. Clearly, under the average power constraint, we have $P_{\mathrm{av} H}=P_{\mathrm{av} M}$ and $A_{H}^{2}=A_{M}^{2} \cdot n_{M} / n_{H}$. Also, it should be noticed that with same transmission rates for both hybrid and ordinary schemes, the average power constraint implies transmission of same energy per bit. In this case, the average received signal-to-noise ratio for both schemes is given by

$$
\mathrm{SNR}_{\mathrm{av}}=\frac{P_{\mathrm{av}}}{\sigma_{n}^{2}}=\frac{A_{H}^{2} \cdot n_{H}}{M \sigma_{n}^{2}}=\frac{A_{M}^{2} \cdot n_{M}}{M \sigma_{n}^{2}}
$$

The symbol-error rates achieved by the proposed hybrid scheme and the ordinary MPPM schemes (both coherent and direct detection) are plotted versus the average signal-to-noise ratio $\left(\mathrm{SNR}_{\mathrm{av}}\right)$ in Figs. 10 and 11 for the cases $M=12$ and $M=24$, respectively. From these figures it is clear that at all levels of $\mathrm{SNR}_{\mathrm{av}}$, the hybrid schemes outperform their MPPM counterparts for both coherent and direct detection schemes. Numerically, as indicated in Fig. 10, at $\mathrm{SNR}_{\mathrm{av}}=10 \mathrm{~dB}$, the hybrid 2-BPSK 12-PPM scheme achieves more than two order of magnitude less SER than those of the coherent detection and direct detection 3-pulse 12-PPM schemes. Also as expected, the coherent detection MPPM schemes have better performance than that of the direct detection ones.

Furthermore, the performance gap between the hybrid schemes and ordinary ones is significantly increased by raising the level of $\mathrm{SNR}_{\mathrm{av}}$. Specifically, for coherent 3-pulse 12-PPM 


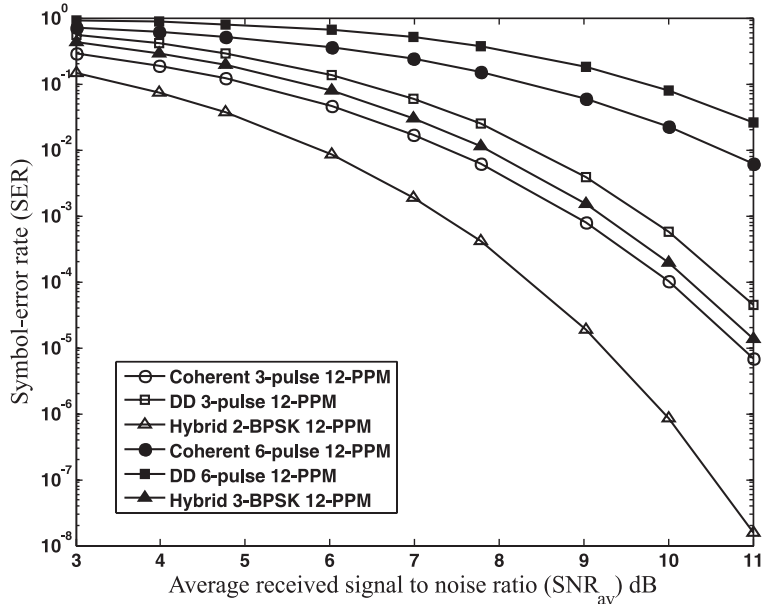

Fig. 10. Symbol-error rate versus average received signal-to-noise ratio at $M=12$.

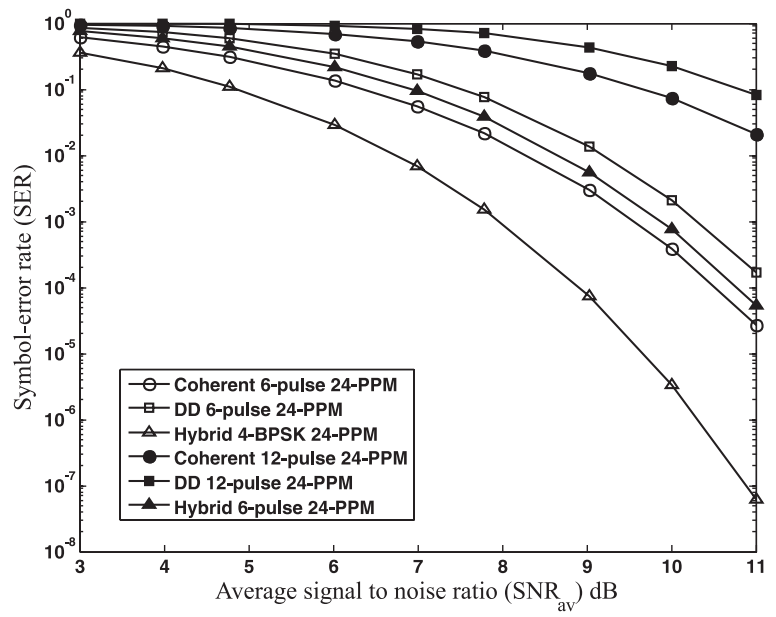

Fig. 11. Symbol-error rate versus average received signal-to-noise ratio at $M=24$.

and hybrid 2-BPSK 12-PPM schemes, raising the $\mathrm{SNR}_{\mathrm{av}}$ level from 6 to $8 \mathrm{~dB}$ nearly doubles the performance gap between both schemes. This may be interpreted as follows. With same average receiving power, raising the $\mathrm{SNR}_{\mathrm{av}}$ level results in noticeable increasing in the received pulse power for the hybrid scheme over the ordinary ones which in turn leads to better signal detection and larger performance gap. Also as expected, increasing the number of optical pulses per frame degrades the achieved performance. Obviously, this comes from the fact that with the same $\mathrm{SNR}_{\mathrm{av}}$ level increasing number of optical pulses per frame reduces the received slot power, which in turn decreases the capability of correct decoding.

Moreover, with the same number of optical pulses per frame, increasing the frame size would result in better performance. It is clear from Figs. 10 and 11 that, at the same levels of $\mathrm{SNR}_{\mathrm{av}}$, the performance of the hybrid 4-BPSK 24-PPM scheme (worse than that of the hybrid 3-BPSK 24-PPM scheme) is always better than that of the hybrid 3-BPSK 12-PPM scheme. This is because with same $\mathrm{SNR}_{\mathrm{av}}$ level and same number of optical pulses per frame, increasing the frame size from 12 to 24 slots results in doubling the pulse power, which in turn increases the probability of correct decoding for the transmitted frame.

\section{CONCLUSION}

A new hybrid modulation scheme based on combining both BPSK and MPPM techniques has been proposed. The scheme has been called hybrid hybrid BPSK-modified MPPM. The aim of the proposed scheme is to enhance the performance of both ordinary BPSK modulation and ordinary MPPM techniques in optical fiber communications systems. The transmitter and receiver block diagram, the transmission characteristics, and the optimal decoding have been studied for the proposed scheme. The performance measure of the proposed scheme in terms of exact symbol-error rate (SER) has been derived taking into account the effect of the ASE noise. An upper bound on the biterror rate (BER) has been obtained for the proposed scheme, as well. Extensive numerical evaluations have been performed to compare the performance achieved by the proposed hybrid scheme to that of ordinary BPSK and MPPM schemes. Our results reveal that under average power constraint, the proposed modulation technique outperforms ordinary MPPM (both coherent and direct detected) scheme at all values of signal-to-noise ratio and outperforms ordinary BPSK at moderate and high levels of signal-to-noise ratios (which are of practical interest). In addition, the proposed scheme achieves much higher bandwidth utilization efficiency than that of the ordinary schemes. However, as a future research work and as an extension to the current research, both polarization-diversity coherent detection and polarization division multiplexing could be considered. Specifically, the hybridization between PM-QPSK and MPPM could be considered.

\section{REFERENCES}

[1] K.-P. Ho, Phase-Modulated Optical Communication Systems. New York, NY, USA: Springer-Verlag, 2005.

[2] D. Slepian, "Permutation modulation," Proc. IEEE, vol. 53, no. 3, pp. 228 236, Mar. 1965.

[3] H. Sugiyama and K. Nosu, "MPPM: A method for improving the bandutilization efficiency in optical PPM," J. Lightw. Technol., vol. 7, no. 3, pp. 465-471, Mar. 1989.

[4] J. Singh and V. K. Jain, "Performance analysis of BPPM and M-ary PPM optical communication systems in atmospheric turbulence," IETE Tech. Rev., vol. 25, no. 4, pp. 146-153, Jul./Aug. 2008.

[5] M. Karlsson and E. Agrell, "Multilevel pulse-position modulation for optical power-efficient communication," Opt. Exp., vol. 19, no. 26, pp. B799B804, 2011.

[6] G. E. Atkin and K. S. Fung, "Performance analysis of coded optical PPM system using direct and coherent detection," IEEE Proc. I: Commun., Speech Vis., vol. 137, no. 4, pp. 226-232, Aug. 1990.

[7] M. M. Fernández and V. A. Vilnrotter. "Performance analysis and preliminary experimental verification of a coherent optical receiver for PPM signals in the presence of atmospheric turbulence," Pasadena, CA, USA: Jet Propuls. Lab., Nat. Aeron. Space Administ., Tech. Rep., Mar. 2004

[8] V. A. Vilnrotter and C. Lau, "Quantum detection and channel capacity for communications applications," Proc. SPIE, vol. 4635, pp. 103-115, 2002.

[9] M. Faridzadeh, A. Gholami, Z. Ghassemlooy, and S. Rajbhandari, "Hybrid PPM-BPSK subcarrier intensity modulation for free space optical communications," in Proc. 16th Eur. Conf. Netw. Opt. Commun., Jul. 20-22, 2011, pp. 36-39.

[10] X. Liu, T. H. Wood, R. W. Tkach, and S. Chandrasekhar, "Demonstration of record sensitivity in an optically pre-amplified receiver by combining PDM-QPSK and M-ary pulse-position modulation," J. Lightw. Technol., vol. 30, no. 4, pp. 406-413, Feb. 15, 2012. 
[11] M. Sjödin, T. A. Eriksson, P. A. Andrekson, and M. Karlsson, "Long-haul transmission of PM-2PPM-QPSK at $42.8 \mathrm{Gbit} / \mathrm{s}$," in Proc. Opt. Fiber Commun. 2013, 2013, pp. 1-3.

[12] X. Liu, S. Chandrasekhar, T. H. Wood, R. W. Tkach, P. J. Winzer, E. C. Burrows, and A. R. Chraplyvy, "M-ary pulse-position modulation and frequency-shift keying with additional polarization/phase modulation for high-sensitivity optical transmission," Opt. Exp., pp. B868-B881, 2011.

[13] J. Hamkins and B. Moision, "Multipulse PPM on memoryless channels," in Proc. Int. Symp. Inf. Theory, Chicago, IL, USA, Jun. 2004, p. 336.

[14] S. Liu and F. R. Kschischang, "Coding for MPPM-like systems," presented at the 25th Bienn. Symp. Commun., Kingston, ON, Canada, May 12-14, 2010.

[15] M. Simon and V. Vilnrotter, "Performance analysis and tradeoff for dualpulse PPM on optical communications channels with direct detection," IEEE Trans. Commun., vol. 52, no. 11, pp. 1969-1979, Nov. 2004

[16] N. Aoki, T. Ohtsuki, and I. Sasase, "Performance analysis of multi-pulse pulse position modulation using avalanche photodiode in optical intersatellite links," IEICE Trans. Commun., vol. E79-B, no. 1, pp. 52-56, Jan. 1996.

[17] M. Martinelli and R. A. Chipman, "Endless polarization control algorithm using adjustable linear retarders with fixed axes," J. Lightw. Technol., vol. 21, no. 9, pp. 2089-2096, Sep. 2003.

[18] J. G. Proakis, Digital Communications, 4th ed. Boston, MA, USA: McGraw-Hill, 2001.

[19] A. M. Rocha, P. Antunes, F. Domingues, M. Facao, and P. S. Andre, "Detection of fiber fuse effect using FBG sensors," IEEE Sens. J., vol. 11, no. 6, pp. 1390-1394, Jun. 2011.

[20] S. Benedetto and E. Biglieri, Principles of Digital Transmission: With Wireless Applications. Norwell, MA, USA: Kluwer, 2002.

[21] X. Liu, S. Chandrasekhar, and A. Leven, "Self-coherent optical transport systems," in Opt.Fiber Telecommun. V B, Systems and Networks, I. P. Kaminow, T. Li, and A. E. Willner, Eds. Burlington, MA, USA: Academic, ch. 4, 2008.

Hossam Selmy was born in Giza, Egypt, in 1979. He received the B.S. and M.S. degrees from Cairo University, Cairo Egypt, in 2001 and 2007, respectively, and the Ph.D. degree from Egypt-Japan University for Science and Technology (EJUST), Alexandria, Egypt, in 2013, all in electrical engineering. He is currently a Research Fellow at the Graduate School of Engineering, Egypt-Japan University for Science and Technology (EJUST). His research interests include advanced modulations and multiple access schemes for both optical fiber communications and next generation wireless access networks.
Hossam M. H. Shalaby (S'83-M'91-SM'99) was born in Giza, Egypt, in 1961. He received the B.S. and M.S. degrees from Alexandria University, Alexandria, Egypt, in 1983 and 1986, respectively, and the Ph.D. degree from the Universityof Maryland at College Park in 1991, all in electrical engineering. In 1991, he joined the Department of Electrical Engineering, Alexandria University, and was promoted to Professor in 2001. He is currently on leave from Alexandria University, where he is the Chair of the Department of Electronicsand Communications Engineering, School of Electronics, Communications, and Computer Engineering, Egypt-Japan University of Science and Technology(E-JUST), New Borg EL-Arab City, Alexandria, Egypt. From December 2000 to 2004, he was an Adjunct Professor with the Faculty of Sciences and Engineering, Department of Electrical and Information Engineering, Laval University, Quebec, QC, Canada From September 1996 to February 2001, he was onleave from the Alexandria University. From September 1996 to January 1998, he was with the Department of Electrical and Computer Engineering , International Islamic University Malaysia, and from February 1998 to February 2001, he was with the School of Electrical and Electronic Engineering, Nanyang Technological University, Singapore. His research interests include optical communications, optical CDMA optical burst-switching, OFDM technology, and information theory. Dr. Shalaby has served as a Sudent Branch Counselor at Alexandria University, IEEE Alexandria, and North Delta Subsection, from 2002 to 2006, and served as a Chairman of the student activities committee of IEEE Alexandria Subsection from 1995 to 1996. He received an SRC fellowship from 1987 to1991 (Systems Research Center, Maryland), State Excellence Award in Engineering Sciences in 2007 (Academy of Scientific Research and Technology, Egypt), Shoman Prize for Young Arab Researchers in 2002 from The Abdul Hameed Shoman Foundation, Amman, Jordan; the State Incentive Award inEngineering Sciences both in 1995 and 2001 from the Academy of Scientific Research and Technology, Egypt; the University Excellence Award in 2009from Alexandria University; and the University Incentive Award in 1996 from Alexandria University.

Zen-Ichiro Kawasaki received the B.S., M.S. and Dr. Eng degrees in communication engineering from Osaka University in 1973, 1975, and 1978, respectively. In 1989, he joined the Department of Electrical Engineering, Osaka University At present, he is a Professor of electrical, electronics and information engineering from Osaka University. He is also an Advisor and Dean in Egypt-Japan University for Science and Technology, Alexandria, Egypt. His main research interests include radar based remote sensing, passive and active remote sensing of atmosphere from space born platforms, and atmospheric electricity. 